\title{
Criminologie
}

\section{Bibliographie sélective sur la rééducation}

\section{Vesna Blazina}

Volume 11, numéro 1, 1978

Centres d’accueil du Québec : accréditation et évaluation

URI : https://id.erudit.org/iderudit/017085ar

DOI : https://doi.org/10.7202/017085ar

Aller au sommaire du numéro

Éditeur(s)

Les Presses de l'Université de Montréal

ISSN

0316-0041 (imprimé)

1492-1367 (numérique)

Découvrir la revue

Citer ce document

Blazina, V. (1978). Bibliographie sélective sur la rééducation. Criminologie, 11(1), 80-86. https://doi.org/10.7202/017085ar d'utilisation que vous pouvez consulter en ligne.

https://apropos.erudit.org/fr/usagers/politique-dutilisation/ 
BIBLIOGRAPHIE SÉLECTIVE SUR LA RÉEDUCATION

\section{Vesna Blazina documentaliste du}

GROUPE DE RECHERCHE SUR L'INADAPTATION JUVÉNILE DE L'UNIVERSITÉ DE MONTRÉAL

Dans les pages qui suivent, nous présentons une sélection des publications récentes sur la rééducation. Notre bibliographie comprend 43 documents (livres, thèses et publications officielles), en anglais et en français, publiés entre 1974 et février 1977 ; les articles ont été exclus parce qu'ils ne sont pas directement accessibles à tous et aussi parce qu'ils sont tellement nombreux qu'une sélection est extrêmement difficile. Par notre choix, nous avons voulu refléter les tendances diverses du domaine ainsi que les thèmes privilégiés par la documentation tels que la déinstitutionnalisation et l'intervention en milieu ouvert. Les ouvrages ont été rassemblés selon le type d'approche adopté (ouvrages de synthèse, études empiriques) et selon le sujet dont ils traitent (intervention en milieu fermé, foyers de groupe, intervention en milieu ouvert, récidive). Afin de donner une meilleure idée du contenu du document, chaque référence est suivie de descripteurs (mots clés) utilisés dans l'analyse documentaire au GRIJ. Tous les documents sont disponibles à notre centre de documentation du Groupe de recherche sur l'inadaptation juvénile de l'Université de Montréal.

\section{A) Ouvrages de synthèse}

1. COFFEY, Alan R., Juvenile Corrections: Treatment and Rehabilitation. Englewood Cliffs, N.J., Prentice-Hall, 1975, 288 p. justice pour mineurs, réducation, institutions, personnel.

2. CUSSON, Maurice, la Resocialisation du jeune délinquant, Montréal, P.U.M., 1974, 160 p.

resocialisation, intervention en milieu fermé, psychanalyse, psychoéducation, éducateurs, relations éducateurs-élèves, Boscoville, Mont Saint-Antoine, Québec.

3. ELDEFONSO, Edward, HARTINGER, Walter, Control, treatment, and rehabilitation of juvenile offenders, Beverly Hills, Glencoe Press, 1976, $321 \mathrm{p}$.

justice pour mineurs, tribunal pour mineurs, détention, intervention en milieu fermé, probation, resocialisation, police, Etats-Unis.

4. FOX, Vernon, Community-based corrections, Englewood Cliffs, Prentice-Hall, 1977,320 p.

intervention en milieu ouvert, déjudiciarisation, maisons de transition, foyers de groupe, désinstitutionnalisation, bénévoles, services à la jeunesse, Youth Service Bureau. 
5. LIPTON, Douglas et al., The effectiveness of correctional treatment. $A$ survey of treatment evaluation studies, New York, Praeger, 1975,735 p.

traitement, rééducation, recherche évaluative.

6. PERISTEIN, Gary, PHELPS, Thomas (eds.). Alternatives to prison. Community based corrections. A reader, Pacific Palisades, Ca., Goodyear Publishing, 1975, $370 \mathrm{p}$.

intervention en milieu ouvert, désinstitutionnalisation, réinsertion au travail, services à la jeunesse.

\section{B) Etudes empiriques}

B. 1) Intervention en milieu fermé

7. CARTER, Barbara Lillian, On the grounds: informal culture in a girls reform school, Ph.D. Thesis - Brandeis University, 1972, Ann Arbor, Xerox, 1976, 253 p.

délinquantes mineures, institutions, relations interpersonnelles, vie en institution, carcéralisation, homosexualité, sous-culture, observation participante.

8. CORNISH, D.B., CLARKE, R.V.G., Residential treatment and its effects on delinquency, London, HMSO, 1975, $74 \mathrm{p}$. intervention en milieu fermé, évaluation, récidive, Kingswood Training School-Bristol.

9. CRAIG, Robert Sylvester, Changes in self concepts and academic achievement of institutionalized delinquent boys in a differential treatment program, Ph.D. Thesis (Ed.) - Indiana University, 1975, Ann Arbor, Xerox, 1976, 121 p.

délinquants mineurs institutionnalisés, perception de soi, succès académique, traitement différentiel.

10. DUNLOP, Anne B., The approved school experience. An account of boys experiences of training under differing regimes of approved schools, with an attempt to evaluate the effectiveness of that training, London, HMSO, 1974, 124 p.

institutions, rééducation, formation professionnelle, intervention en milieu fermé, évaluation, Grande-Bretagne.

11. ERICSON, Richard V., Young offenders and their social work, Westmead, England, Saxon House, Lexington, D.C. Heath, 1975, 225 p. détention, carcéralisation, resocialisation, réinsertion sociale, observation participante, Grande-Bretagne.

12. GIALLOMBARDO, Rose, The social world of imprisoned girls. A comparative study of institutions for juvenile delinquents, New York, Toronto, Wiley, 1974, 1974, $317 \mathrm{p}$.

délinquantes mineures, institutions, clientèle, relations interpersonnelles, rôles, vie en institution, carcéralisation, homosexualité, sousculture, études comparées, Etats-Unis.

13. GUINDON, Jeannine, les Etapes de la rééducation des jeunes délinquants et des autres, éd. revue et augmentée, Paris, Fleurus, 1975, $328 \mathrm{p}$.

psycho-éducation, rééducation, éducateurs, Boscoville.

14. LAFLAMME-CUSSON, Suzanne, BARIL, Micheline, la Détention des mineurs dans la région de Montréal, Montréal, ministère des Affaires sociales, 1975. $4 \mathrm{v}$.

centres d'accueil, clientèle, services à la jeunesse, prisons, cellules de la police, Montréal, St-Vallier, Villa Notre-Dame de Grâce, Franc-Bord, Berthelet. 
15. MENARD, Robert, le Climat social dans une institution pour jeunes délinquants: Boscoville, Rapport technique no 13, Montréal, G.R.I.J., 1976, 290 p.

climat social, instruments de mesure, Boscoville.

16. MONTARON. Jean-Pierre, les Jeunes en prison, Paris, Seuil, 1977, 253 p.

jeunes adultes délinquants, récits, France.

17. QUEBEC, Ministère des Affaires sociales, Comité Batshaw, Rapport du Comité d'étude sur la réadaptation des enfants et adolescents placés en centre d'accueil, Soumis au ministère des Affaires sociales du Québec, le 22 décembre 1975. Québec, 1976, 174 p. centres d'accueil, centres de réadaptation, Québec.

18. SARRI, Rosemary C., Under lock and key: juveniles in jails and detention, Ann Arbor, National Assessment of Juvenile Corrections, 1974,85 p.

détention, délinquants mineurs, prisons, clientèle, Etats-Unis.

19. SCHOUTEN, Jan et autres, Garde ton masque. Traitement résidentiel des adolescents: l'expérience de Zandwijk (Amersfoort), Paris, Fleurus, 1976, 351 p. Traduit du néerlandais : Laat je niet kennen (1974).

intervention en milieu fermé, thérapie de groupe, thérapie familiale, thérapie transactionnelle, éducateurs, formation professionnelle, personnalité délinquante, Pays-Bas.

20. SIGLER, Robert Thomas, Influence and gang membership in three juvenile correctional institutions, Ph.D. Thesis - University of Missouri, 1974, Ann Arbor, Xerox, 1976, 219 p.

sociométrie, meneurs, bandes de jeunes, institutions, Illinois.

21. TIZARD, Jack et al. (eds.), Varieties of residential experience, LonCon, Routledge \& Kegan Paul, 1975, 290 p.

intervention en milieu fermé, institutions, délinquants mineurs, inadaptés sociaux, arriérés mentaux, socialisation, fugue, études comparées, Grande-Bretagne.

22. TREMBLAY, Richard Ernest, $A$ psycho-educational study of juvenile delinquents during residential treatment. Ph.D. Thesis (Ed.) University of London, $1976,302 \mathrm{p}$.

psycho-éducation, resocialisation, intervention en milieu fermé, climat social, comportement, études empiriques, études longitudinales, Londres, Québec.

23 TUTT, Norman, Care or custody. Community homes and the treatment of delinquency, London, Darton, Longman and Todd, 1974, 228 p.

intervention en milieu fermé, Wellside School, Grande-Bretagne.

24. VINTER, Robert D. et al. (eds.), Time out. A national study of juvenile correctional programs, Ann Arbor, National Assessment of Juvenile Corrections, University of Michigan, 1976, $250 \mathrm{p}$. intervention en milieu fermé, intervention en milieu ouvert, institutions, délinquants mineurs, enquêtes par questionnaires, Etats-Unis.

25. VODOPIVEC, Katja et al. (eds.), Maladjusted youth. An experiment in rehabilitation, Westmead, England, Saxon House, Lexington (Mass.), Lexington Books, D.C. Heath, 1974, 275 p.

intervention en milieu fermé, rééducation, éducateurs, formation professionnelle, thérapie de groupe, personnalité, Yougoslavie. 


\section{B. 2) Foyers de groupe}

26. BLUM, Dorothy Jean Scrivner, Case studies in an evaluation of a community based home for juvenile delinquents, Ph.D. Thesis (Ed.) University of Virginia, 1975, Ann Arbor, Xerox, 1976, 254, A-52, B-60, C-26, D-2p.

foyers de groupe, évaluation, études des cas, maturité interpersonnelle, Community Attention Home - Charlottesville, Virginie.

27. COLLEGE OF WILLIAM AND MARY, Metropolitan Criminal Justice Center. Juvenile group homes in the city of Norfolk, Virginia. A status report, Williamsburg (Va.), 1974, 64 p. foyers de groupe, Norfolk, Virginie.

28. DUSSAULT, Anne, LEBLANC, Marc, Evaluation des foyers de groupe, Montréal, GRIJ, 1976, 137 p.

foyers de groupe, évaluation, Québec.

Cet ouvrage comprend une bibliographie exhaustive sur les foyers de groupe en Amérique du Nord.

29. KIRIGIN, Kathryn A., FIXSEN, Dean L., The evaluation of six group homes in Kansas, Lawrence, Kansas, Bureau of Child Research, 1974, 73 p.

foyers de groupe, évaluation, récidive, succès académique, coût, Kansas.

30. NATIONAL COUNCIL ON CRIME AND DELINQUENCY, Survey and Planning Center. The Maryland group home program. Innovative substitute for incarceration of children. Recommendations - standards, Austin, 1974, 193, A-19, B-16, C-18p.

foyers de groupe, normes, Maryland.

31. PHILLIPS, Elery L. et al., The teaching-family handbook: group living environments administered by professional teaching-parents for youths in trouble, Lawrence, Kansas, University of Kansas, 1972-1974, 384 p.

foyers de groupe, renforcement positif, modification comportementale, éducateurs, Achievement Place.

B. 3) Intervention en milieu ouvert

32. LATHAN, Alfonza, Community transition in youth rehabilitation, Ph.D. Thesis - University of New Mexico, 1976, Ann Arbor, Xerox, 1977,110 p.

intervention en milieu ouvert, désinstitutionnalisation, récidive, Massachusetts.

33. LERMAN, Paul, Community treatment and social control. A critical analysis of juvenile correctional policy, Chicago, University of Chicago Press, 1975, 254 p.

intervention en milieu ouvert, maturité interpersonnelle, probation, évaluation, Community Treatment Project — Californie.

34. MILLER, Mercedese M. (ed.), Evaluating community treatment proggrams. Tools, techniques and a case study, Lexington, Toronto, D.C. Heath, 1975,124 p.

intervention en milieu ouvert, maisons de transition, évaluation, études des cas, Congress Heights Human Development Center Washington, D.C.

35. OHLIN, Lloyd E. et al., Juvenile correctional reform in Massachusetts, Washington, Law Enforcement Assistance Administration, 1977, 322 p.

intervention en milieu ouvert, désinstitutionnalisation, réforme, foyers de groupe, récidive, évaluation, Massachusetts. 
36. ONTARIO, Ministry of Correctional Services, Planning and Research Branch, $A$ review of alternatives to the incarceration of the youthful offender. Toronto, 1976, $80 \mathrm{p}$.

désinstitutionnalisation, intervention en milieu ouvert, foyers de groupe, traitement différentiel, services à la jeunesse.

37. VINTER, Robert D. et al., Juvenile corrections in the States: residential programs and deinstitutionalization. A preliminary report, Ann Arbor, National Assessment of Juvenile Corrections, University of Michigan, $1976,80 \mathrm{p}$.

désinstitutionnalisation, intervention en milieu ouvert, Etats-Unis.

B. 4) Récidive et réinsertion sociale

38. BEAULNE, A., le Devenir social des anciens de Boscoville, Montréal, G.R.I.J., 1976, 201 p.

réinsertion sociale, vécu post-institutionnel, perception de soi, personnalité, relations interpersonnelles, réinsertion au travail, études follow-up, Boscoville.

39. FISHMAN, Gideon, Post-institutional outcome and functioning : a study of juvenile residential treatment center, Ph.D. Thesis - Ohio State University, 1974, Ann Arbor, Xerox, 1975, 180 p.

intervention en milieu fermé, évaluation, réinsertion sociale, récidive, délinquance révélée, études follow-up, Buckeye Boys Ranch - Ohio.

40. GOODMAN, Nancy et al., Further studies of female offenders. Part A. Borstal girls eight years after release. Part B. The sentencing of women at the London higher courts. Part C. Girls appearing before a juvenile court, London, HMSO, 1976, $111 \mathrm{p}$.

délinquance féminine, études follow-up, récidive, réinsertion sociale, tribunal pour mineurs, milieu familial.

41. LORD, John Harlan., Predicting recidivists and non-recidivists in a delinquent population, Ph.D. Thesis - University of Maryland, 1974, Ann Arbor, Xerox, 1976, 125 p. prévision, récidive, personnalité, délinquants mineurs institutionnalisés.

42. PETITCLERC, Nicole, Etude follow-up à Boscoville, Montréal, G.R.I.J., 1974, 129 p. récidive, études follow-up, Boscoville.

43. SAWYER, Melvin Wayne, The effects of community probation unit services versus conventional probation services on recidivism by juvenile probationers, Ph.D. Thesis - Brigham Young University, 1975. Ann Arbor, Xerox, 1976, 78 p.

récidive, probation, sexe, âge, délinquance statutaire. 


\section{INDEX}

Achievement Place 31

Âge 43

Arriérés mentaux 21

Bandes de jeunes 20

Bénévoles 4

Berthelet 14

Boscoville 2, 13, 15, 38, 42

Buckeye Boys Ranch - Ohio 39

Carcéralisation 7, 11, 12

Cellules de la police 14

Centres d'accueil 14, 17

Centres de réadaptation 17

Clientèle 12, 14, 18

Climat social 15, 22

Community Attention Home Charlottesville, Virginie 26

Community Treatment Project Californie 33

Comportement 22

Congress Heights Human Development Center - Washington, D.C. 14

Coût 29

Désinstitutionnalisation $4,6,32,35$, 36,37

Déjudiciarisation 4

Delinquance féminine 40

Delinquance révélée 39

Délinquance statutaire 43

Délinquantes mineures 7,12

Délinquants mineurs $18,21,24$

Délinquants mineurs institutionnalisés 9,41

Détention 3, 11, 18

Éducateurs 2, 13, 19, 25, 31

Enquêtes par questionnaires 24

Etats-Unis 3, 12, 18, 24, 37

Études comparées 12, 21

Etudes des cas 26, 34

Études empiriques 22

Études follow-up $38,39,40,42$

Études longitudinales 22

Evaluation 8, 10, 26, 28, 29, 33, 34, 35,39

Formation professionnelle 10, 19, 25

Foyers de groupe $4,26,27,28,29$, $30,31,35,36$

Franc-Bord 14

France 16

Fugue 21

Grande-Bretagne 10, 11, 21, 23

Homosexualité 7, 12

Illinois $\mathbf{2 0}$
Inadaptés sociaux 21

Institutions 1, 7, 10, 12, 20, 21, 24

Instruments de mesure 15

Intervention en milieu fermé $2,3,8$, $10,20,21,22,23,24,25,39$

Intervention en milieu ouvert 4,6 , $24,32,33,34,35,36,37$

Jeunes adultes délinquants 16

Justice pour mineurs 1,3

Kansas 29

Kingswood Training School Bristol 8

Londres 22

Maisons de transition 4, 34

Maryland 30

Massachusetts 32, 35

Maturité interpersonnelle 26, 33

Meneurs 20

Milieu familial 40

Modification comportementale 31

Montréal 14

Mont Saint-Antoine 2

Norfolk - Virginie 27

Normes 30

Observation participante 7, 11

Pays-Bas 19

Perception de soi 9, 38

Personnalité 25, 38, 41

Personnalité délinquante 19

Personnel 1

Police 3

Prévision 41

Prisons 14, 18

Probation 3, 33, 43

Psychanalyse 2

Psycho-éducation 2, 13, 22

Québec 2, 17, 22, 28

Recherche évaluative 5

Récidive $8,29,32,35,39,40,41$, 42,43

Récits 16

Rééducation 1, 5, 10,13, 25

Réforme 35

Réinsertion au travail 6, 38

Réinsertion sociale $11,38,39,40$

Relations éducateurs-élèves 2

Relations interpersonnelles 7, 12, 38

Renforcement positif 31

Resocialisation 2, 3, 11, 22

Rôles 12

St-Vallier 14

Services à la jeunesse 4, 6, 14, 36

Sexe 43 
Socialisation 21

Sociométrie 20

Sous-culture 7, 12

Succès académique 9,29

Thérapie de groupe 19,25

Thérapie familiale 19

Thérapie transactionnelle 19

Traitement 5
Traitement différentiel 9,36

Tribunal pour mineurs 3,40

Vécu post-institutionnel 38

Vie en institution 7, 12

Villa Notre-Dame de Grâce 14

Wellside School 23

Yougoslavie 25

Youth Service Bureau 4 